\title{
Infantile hemangioma is a proliferation of LYVE-1-negative blood endothelial cells without lymphatic competence
}

\author{
Van An Nguyen ${ }^{1}$, Heinz Kutzner ${ }^{2}$, Christina Fürhapter ${ }^{1}$, Alexandar Tzankov ${ }^{3}$ and \\ Norbert Sepp ${ }^{1}$ \\ ${ }^{1}$ Department of Dermatology, Innsbruck Medical University, Innsbruck, Austria; ${ }^{2}$ Dermatopathologisches \\ Gemeinschaftslabor, Friedrichshafen, Germany and ${ }^{3}$ Department of Pathology, Innsbruck Medical University, \\ Innsbruck, Austria
}

\begin{abstract}
Infantile hemangiomas are common benign vascular tumors that exhibit a characteristic history of rapid proliferation in the first year of life and slow spontaneous involution during early childhood. The causative pathogenic event responsible for the abnormal endothelial proliferation remains elusive. The recent discovery of an immature phenotype of proliferating hemangioma endothelial cells due to the exclusive expression of the lymphatic endothelial hyaluronan receptor LYVE-1 led to the proposal that infantile hemangiomas are the result of a primary defect in endothelial cell maturation. To test this hypothesis, we looked for the expression of the lymphatic endothelial cell-specific markers LYVE-1, Prox-1, podoplanin and D2-40 in $\beta 4$ integrin-negative proliferating and $\beta 4$ integrin-positive involuting infantile hemangiomas. As $\beta 4$ integrin proved to be a suitable marker for staging infantile hemangiomas, we used it in combination with clinical and histological criteria to objectively determine the proliferative and involutional phases. In immunohistochemical and immunofluorescent stains, hemangioma vessels were negative for all lymphatic endothelial cell-specific markers tested during both proliferation and involution. LYVE-1 immunoreactivity, however, was found in the dense network of perivascular HLA-DR-positive cells with dendritic cell morphology that are supposed to play a role in hemangiogenesis by releasing pro- and antiangiogenic factors. Notably, this LYVE-1 staining failed to correlate with the growth status of infantile hemangiomas. Our results do not support the notion that LYVE-1 expression was restricted to the proliferative phase and downregulated during involution. Thus, LYVE-1 does not seem to be a reliable marker for proliferating infantile hemangiomas. We conclude that the suggested intrinsic defect in endothelial cell maturation is unlikely the cause for the post-natal rapid growth in infantile hemangiomas. In addition, the lack of lymphatic endothelial cell-specific markers implies that infantile hemangiomas are tumors of blood vessels without lymphatic competence.
\end{abstract}

Modern Pathology (2006) 19, 291-298. doi:10.1038/modpathol.3800537

Keywords: infantile hemangioma; proliferation; involution; lymphatic specific endothelial cell markers

Infantile hemangiomas are endothelial tumors with an estimated incidence of up to $10 \%$, making them the most common tumor of infancy. ${ }^{1,2}$ The biologic behavior of this benign angiomatous disorder is characterized by rapid growth during the first year of post-natal life (proliferative phase), followed by slow spontaneous regression over a period of several years (involutional phase). ${ }^{3}$ Using immunohistochemistry on frozen sections, we recently identified

Correspondence: Dr VA Nguyen, MD, Department of Dermatology, Innsbruck Medical University, Anichstrasse 35, A-6020 Innsbruck, Austria.

E-mail: van.nguyen@uibk.ac.at

Received 19 September 2005; revised 10 November 2005; accepted 14 November 2005 $\beta 4$ integrin as a novel marker for staging infantile hemangiomas, showing that $\beta 4$ integrin was exclusively expressed in regressing and involuting infantile hemangiomas, but was lacking in proliferating infantile hemangiomas. ${ }^{4}$

Although infantile hemangiomas are well-known vascular lesions, the cellular and molecular mechanisms that cause the abnormal excessive endothelial cell proliferation are still poorly understood. Many theories have been proposed concerning the pathogenesis of infantile hemangiomas, including hormonal influences and growth factors that are released by adjacent hyperplastic epidermis, neighboring stromal cells and/or hemangioma endothelial cells.5,6 Because infantile hemangiomas share immunophenotypic similarities with fetal placental 
vasculature, some authors argue that they are of placental origin. ${ }^{7}$ Others, in turn, claim they are due to clonal expansion of endothelial cells in which somatic mutations of one or more genes regulating endothelial cell growth have occurred..$^{8,9}$ Equally important was our finding that hemangioma vessels are closely surrounded by densely packed HLA-DR-positive cells with dendritic cell morphology. ${ }^{4}$ This close anatomic relationship to each other prompted us to suggest that infantile hemangiomas are the result of inappropriate vasculogenesis rather than of angiogenesis. ${ }^{10}$ The latter is currently regarded as the critical inductive mechanism for the abnormal vessel formation. Most recently, Dadras et $a l^{11}$ detected the specific expression of the lymphatic endothelial hyaluronan receptor LYVE-1 in endothelial cells of proliferating infantile hemangiomas, whereas it was downregulated or absent from lesional capillaries during the involutional phase. Based on the coexpression of LYVE-1 and CD34, which reflects an immunophenotype similar to that of the embryonic vein during normal vascular development, they hypothesized that hemangioma endothelial cells are arrested in an early developmental vascular differentiation state. ${ }^{12}$ In their opinion, this concept of an immature phenotype explains the rapid growth of infantile hemangiomas during the first few months of life.

In the present study, we aimed to test this concept and investigated the expression of the lymphatic endothelial cell-specific markers LYVE-1, Prox-1, podoplanin and D2-40 during the consecutive growth phases of infantile hemangiomas by using immunohistochemistry and immunofluorescence assays. The classification of the growth status into proliferative and involutional phases basically re- lied on the absence and presence of $\beta 4$ integrin, respectively.

\section{Materials and methods}

\section{Patients}

All 18 cases, diagnosed as infantile hemangiomas from 1994 to 2003, were retrieved from the archived files of the Departments of Pathology (15 cases) and Dermatology (two cases), University Innsbruck, Austria, and Dermatohistopathologisches Gemeinschaftslabor (one case), Friedrichshafen, Germany. Diagnosis was confirmed by histological review of hemotoxylin- and eosin-stained slides. Relevant clinical data of the included patients such as gender (sex ratio F:M 2:1), age at the time of resection (mean age 1 year) and the location of the lesions are given in Table 1. On the basis of immunohistochemical staining with antibodies recognizing the $\beta_{4}$ integrin epitope in correlation with clinical history and histological criteria, tissue sections were subdivided into proliferating ( $n=9$, mean age 6.5 months) and involuting $(n=9$, mean age 14 months) infantile hemangiomas, respectively.

\section{Immunohistochemical Analyses}

Formalin-fixed, paraffin-embedded tumor tissues underwent immunohistochemical staining with a wide panel of commercially available antibodies. The antibodies employed in the immunostaining procedures, their sources and dilutions are listed in Table 2. Antigen retrieval by means of a pressure cooker, with or without pronase, was applied.

Table 1 Clinical data of patients

\begin{tabular}{|c|c|c|c|c|c|}
\hline Patient & Sex/age (years) & Location of biopsy & Hemangioma type & Size $(\mathrm{cm})$ & Status of hemangioma \\
\hline 1 & M/0.11 & Frontal & Capillary & 0.9 & Proliferation ${ }^{\mathrm{a}}$ \\
\hline 2 & $\mathrm{~F} / 1.8$ & Nasolabial & Capillary & 1.8 & Proliferation \\
\hline 3 & $\mathrm{~F} / 0.3$ & Occipital & Capillary & 1.3 & Proliferation \\
\hline 4 & $\mathrm{~F} / 0.9$ & Flank & Capillary & 2 & Proliferation \\
\hline 5 & $\mathrm{~F} / 0.4$ & Supraorbital & Capillary & 1.2 & Involution ${ }^{\mathrm{b}}$ \\
\hline 6 & $\mathrm{~F} / 1.7$ & Nasal & Capillary & 0.8 & Involution \\
\hline 7 & $\mathrm{~F} / 0.8$ & Occipital & Capillary & 2.5 & Involution \\
\hline 8 & $\mathrm{~F} / 1.4$ & Labial & Capillary & 3.1 & Proliferation \\
\hline 9 & $\mathrm{~F} / 2.3$ & Chest & Capillary & 3 & Involution \\
\hline 10 & $\mathrm{~F} / 2.2$ & Frontal & Capillary & 1.2 & Involution \\
\hline 11 & $\mathrm{~F} / 1.1$ & Unknown & Capillary & 4 & Involution \\
\hline 12 & $\mathrm{~F} / 0.4$ & Chest & Capillary & 2.1 & Proliferation \\
\hline 13 & $\mathrm{M} / 0.7$ & Frontal & Capillary & 1 & Proliferation \\
\hline 14 & $\mathrm{M} / 1.7$ & Popliteal & Capillary & 7 & involution \\
\hline 15 & $\mathrm{M} / 0.9$ & Occipital & Capillary & 1 & Proliferation \\
\hline 16 & $\mathrm{M} / 1.6$ & Nasal & Capillary & 3.4 & Involution \\
\hline 17 & $\mathrm{M} / 0$ & Labial & Capillary & 1 & Proliferation \\
\hline 18 & $F / 2$ & Supraorbital & Capillary & 2 & Involution \\
\hline
\end{tabular}

${ }^{\text {a }}$ Proliferation was clinically defined as an increase in the size of hemangiomas by at least $50 \%$ within the first 3 months of life and histologically characterized by tightly packed small $\beta 4$-negative hemangiomatous capillaries.

${ }^{\mathrm{b}}$ Involution was clinically defined as a reduction in the size of hemangiomas associated with slightly atrophic skin changes and histologically characterized by more remote enlarged $\beta 4$-positive vessels. 
Table 2 Monoclonal and polyclonal antibodies used for immunohistochemical and immunofluorescence stainings

\begin{tabular}{|c|c|c|c|c|c|}
\hline Antibody & $\begin{array}{l}\text { Antigen } \\
\text { retrieval }\end{array}$ & Clone & Isotype & Dilution & Source \\
\hline CD1a & & HI149 & $\operatorname{IgG1}$ & $1: 5$ & BD Pharmingen, San Diego, CA, USA \\
\hline \multirow[t]{2}{*}{ CD31 } & \multirow[t]{2}{*}{$\mathrm{pH} 6.1$} & JC70A & IgG1 & $1: 50$ & Dako, Glostrup, Denmark \\
\hline & & WM-59 & IgG1 & $1: 5$ & BD Pharmingen, San Diego, CA, USA \\
\hline CD34 & \multirow{2}{*}{ pH 6.1} & Q Bend 10 & IgG1 & $1: 5$ & BD Pharmingen, San Diego, CA, USA \\
\hline CD68 & & KP1 & IgG1 & $1: 50$ & Dako, Glostrup, Denmark \\
\hline CD104 ( $\beta 4)$ & \multirow{5}{*}{ pH 6.1} & ELF1 & IgG1 & $1: 50$ & Gibco BRL, Gaithersburg, MD, USA \\
\hline CD207 (Langerin) & & Supplement & IgG1 & Supplement & A generous gift by $\mathrm{K}$ Yoneda \\
\hline CD208 (DC-LAMP) & & & IgG1 & 1.500 & A generous gift by S Lebecque \\
\hline CD209 (DC-SIGN) & & 120507 & IgG1 & $1: 10$ & RD Systems Inc., Tustin, CA, USA \\
\hline BDCA-2 & & AC144 & IgG1 & $1: 10$ & Miltenyi Biotec, Bergisch Gladbach, Germany \\
\hline D2-40 & pH 6.1 & $\mathrm{D} 2-40$ & IgG1 & $1: 100$ & Dako, Glostrup, Denmark \\
\hline \multirow[t]{2}{*}{ GLUT1 } & \multirow[t]{2}{*}{$\mathrm{pH} 6.1$} & Polyclonal & Rabbit serum & $1: 200$ & Abcam Ltd, Cambridge, UK \\
\hline & & Polyclonal & Rabbit serum & $1: 50$ & Abcam Ltd, Cambridge, UK \\
\hline \multirow[t]{2}{*}{ HLA-DR } & \multirow[t]{2}{*}{$\mathrm{pH} 6.1$} & TAL.1B5 & IgG1 & $1: 100$ & Dako, Glostrup, Denmark \\
\hline & & G46-6 & IgG1 & $1: 5$ & BD Pharmingen, San Diego, CA, USA \\
\hline \multirow[t]{2}{*}{ LYVE-1 } & \multirow[t]{2}{*}{ pH 6.1} & Polyclonal & Rabbit serum & $1: 400$ & DCS, Hamburg, Germany \\
\hline & & Polyclonal & Rabbit serum & $1: 100$ & $\begin{array}{l}\text { RDI, Research Diagnostics Inc., Flanders, } \\
\text { Netherlands }\end{array}$ \\
\hline \multirow{2}{*}{$\begin{array}{l}\alpha \text {-smooth muscle } \\
\text { actin }\end{array}$} & \multirow[t]{2}{*}{$\mathrm{pH} 6.1$} & $1 \mathrm{~A} 4$ & IgG1 & $1: 300$ & Dako, Glostrup, Denmark \\
\hline & & $1 \mathrm{~A} 4$ & IgG1 & $1: 100$ & Dako, Glostrup, Denmark \\
\hline \multirow{2}{*}{ Podoplanin } & \multirow{2}{*}{ pH 9.0} & Polyclonal & IgG1 & $1: 200$ & Acris, Hiddenhausen, Germany \\
\hline & & gp36 & IgG1 & $1: 100$ & $\begin{array}{l}\text { RDI, Research Diagnostics Inc., Flanders, } \\
\text { Netherlands }\end{array}$ \\
\hline \multirow[t]{2}{*}{ Prox-1 } & \multirow{2}{*}{ pH 6.1+pronase } & Polyclonal & Rabbit serum & $1: 200$ & RELIA Tech, Braunschweig, Germany \\
\hline & & Polyclonal & Rabbit serum & $1: 100$ & $\begin{array}{l}\text { RDI, Research Diagnostics Inc., Flanders, } \\
\text { Netherlands }\end{array}$ \\
\hline \multirow{2}{*}{$\begin{array}{l}\text { von Willebrand } \\
\text { factor (FVIII) }\end{array}$} & \multirow[t]{2}{*}{ pH 6.1+pronase } & F8/86 & Igg1 & $1: 50$ & Dako, Glostrup, Denmark \\
\hline & & Polyclonal & Rabbit serum & $1: 100$ & Dako, Glostrup, Denmark \\
\hline
\end{tabular}

Appropriate positive and negative controls were used in each case. Immunohistochemical staining was performed by a labeled streptavidin-biotin (LSAB) technique on a robotic stainer (DAKO TechMate 500). Histological slides were coverslipped with Pertex for optimal visual detection of staining results.

\section{Immunofluorescence Analyses}

Cryosections ( $5 \mu \mathrm{m})$ were air-dried, fixed in acetone for $10 \mathrm{~min}$ and then incubated in sequence with primary antibody (see Table 2), biotinylated antimouse Ig (Amersham-Pharmacia, Amersham, UK), and Texas Red-conjugated streptavidin (Amersham). After blocking of residual binding sites with an excess of mouse gamma globulin $(100 \mu \mathrm{g} / \mathrm{ml})$, sections were counterstained with FITC-conjugated secondary antibody. The specimens were mounted in Vectashield (Vector, Burlingame, CA, USA) and viewed on a conventional fluorescence microscope.

\section{Results}

Of 18 specimens, nine were classified as proliferating infantile hemangiomas and nine as involuting infantile hemangiomas. Histologically, proliferation was characterized by tightly packed small heman- gioma capillaries and involution by more remote enlarged vessels instead. Furthermore, involuting infantile hemangiomas expressed $\beta_{4}$ integrin, but proliferating infantile hemangiomas did not.

In all cases, tumor endothelial cells showed intense staining for the panendothelial marker CD31, the blood vascular-specific marker CD34, von Willebrand factor FVIII and, except for patients 13 and 14, the erythrocyte-type glucose transporter protein GLUT-1 (Table 3). Typically, hemangioma vessels were covered by a layer of smooth muscle actin-positive pericytes. As expected, expression of LYVE-1 was absent in involuting infantile hemangiomas. We were, however, surprised by the finding that proliferating infantile hemangiomas also were virtually completely devoid of LYVE-1, with the exception of patient 12, who displayed LYVE-1 positivity in the majority of lesional capillaries (Figure 1a and b). Moreover, lobules of all examined vascular tumors, both proliferating and involuting, were constantly negative for the lymphatic endothelial cell-specific markers Prox-1, podoplanin and D2-40 (Figure 1e, g and h). Results obtained from double immunofluorescence staining with LYVE-1, Prox-1, podoplanin and CD31 were similar to those obtained from single-marker immunohistochemical staining: tumor endothelial cells were positive for CD31, but negative for LYVE-1, Prox-1 and podoplanin (Figure $2 \mathrm{a}-\mathrm{C}$ and $\mathrm{j}-\mathrm{O}$ ). In addition, no 
Table 3 Expression of surface molecules of skin biopsy specimens from hemangioma patients

\begin{tabular}{|c|c|c|c|c|c|c|c|c|}
\hline Patients & $L Y V E-1$ & Prox1 & Podoplanin & $D 2-40$ & $C D 31$ & CD34 & FVIII & GLUT1 \\
\hline 1 & $-^{\mathrm{a}}$ & - & - & - & + & + & + & + \\
\hline 2 & - & - & - & - & + & + & + & + \\
\hline 3 & - & - & - & - & + & + & + & + \\
\hline 4 & $-^{\mathrm{a}}$ & - & - & - & + & + & + & + \\
\hline 5 & $-^{\mathrm{a}}$ & - & - & - & + & + & + & + \\
\hline 6 & - & - & - & - & + & + & + & + \\
\hline 7 & $-^{\mathrm{a}}$ & - & - & - & + & + & + & + \\
\hline 8 & - & - & - & - & + & + & + & + \\
\hline 9 & - & - & - & - & + & + & + & + \\
\hline 10 & - & - & - & - & + & + & + & + \\
\hline 11 & - & - & - & - & + & + & + & + \\
\hline 12 & + & - & - & - & + & + & + & + \\
\hline 13 & - & - & - & - & + & + & + & - \\
\hline 14 & $-^{\mathrm{a}}$ & - & - & - & + & + & + & - \\
\hline 15 & - & - & - & - & + & + & + & + \\
\hline 16 & - & - & - & - & + & + & + & + \\
\hline 17 & $-^{\mathrm{a}}$ & - & ND & ND & + & + & + & + \\
\hline 18 & $-^{\mathrm{a}}$ & - & ND & ND & + & + & + & + \\
\hline
\end{tabular}

Staining was graded - (absent), + (present); ND: not done.

${ }^{\text {a } L Y V E-1-p o s i t i v e ~ p e r i v a s c u l a r ~ c e l l s . ~}$
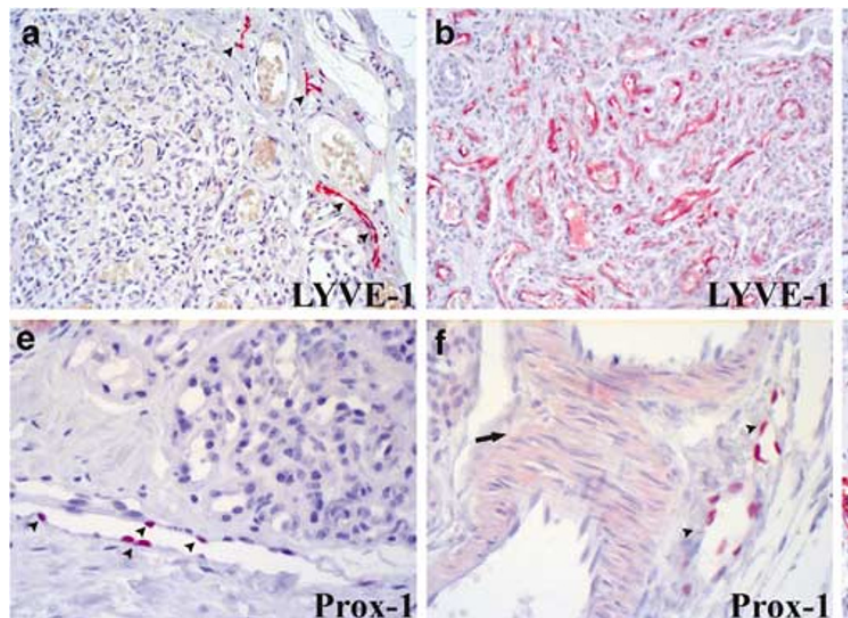
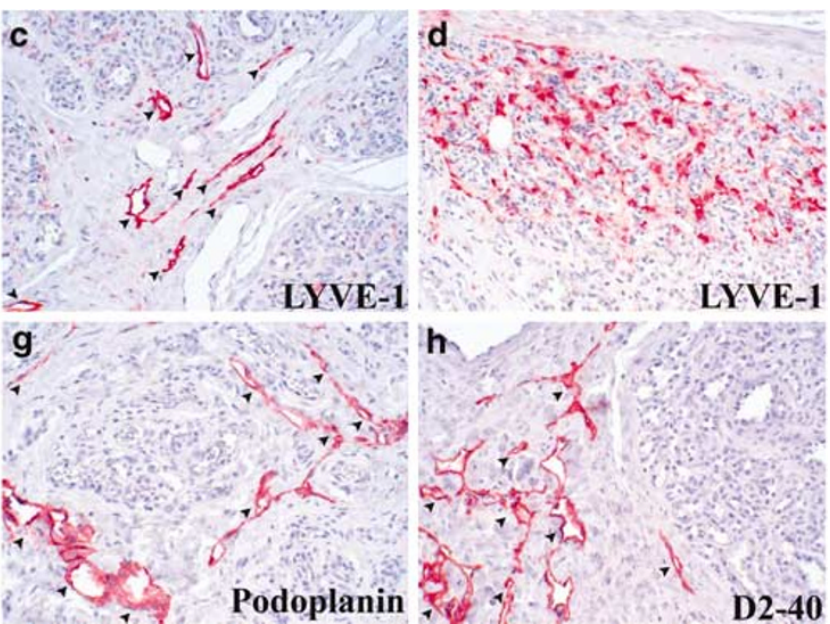

Figure 1 Immunohistochemical staining of proliferating infantile hemangioma tissue with labeled streptavidin-biotin technique. (a) Hemangioma capillaries do not express LYVE-1, (b) except for one case. Note the positive internal controls for LYVE-1 provided by lymphatic vessels at the tumor margin (arrowheads). (c) In contrast, intraseptal lymphatic vessels show strong endothelial immunoreaction for LYVE-1 (arrowheads). (d) In tissue sections of some patients, LYVE-1 ${ }^{+}$cells are detected around hemangioma capillaries. (e, f) Hemangioma endothelial cell nuclei are negative for Prox-1. Also, adjacent blood vascular endothelial cells lack Prox-1 nuclear staining (arrow), whereas lymphatic vessels at the tumor margin and in the tumor septum are immunoreactive for Prox-1 (arrowheads). (g, h) There is no observed immunoreaction for podoplanin and D2-40 in lesional endothelial cells although immunoreactivity for podoplanin and D2-40 is frequently present in interlobular lymphatic vessels (arrowheads). Original magnifications: $\times 200(\mathbf{a}-\mathbf{d}, \mathbf{g}, \mathbf{h}), \times 400(\mathbf{e}, \mathbf{f})$.

staining of blood vessels exhibiting morphological features of arteries or veins with LYVE-1, Prox-1, podoplanin or D2-40 was observed (Figures $1 \mathrm{f}$ and $2 \mathrm{~d}-\mathrm{f}$ ). Contrarily, CD31-positive vessels with large, dilated and thin-walled channels, occasionally located in the vicinity of infantile hemangiomas, coexpressed LYVE-1, Prox-1, podoplanin and D2-40, strongly implying that they were consistent with lymphatic vessels (Figure 1a, c, e-h and $2 \mathrm{~d}-\mathrm{f}$ ). Peritumoral LYVE-1-positive lymphatic vessels were also noted for cases 12-14. LYVE-1 immunoreactivity was further detected in the dense network of intimately surrounding HLA-DR-positive cells that morphologically highly resembled dendritic cells (Figures $1 \mathrm{~d}$ and $2 \mathrm{~g}-\mathrm{i}$ ). Interestingly, overlap of the green HLA-DR and the red LYVE-1 signal was noted only in few patients (patients 1, 4, 5, 14, 17 and 18) and was independent of the growth status of infantile hemangiomas. Immunophenotyping of the HLA-DR-positive cells revealed that CD68 and 

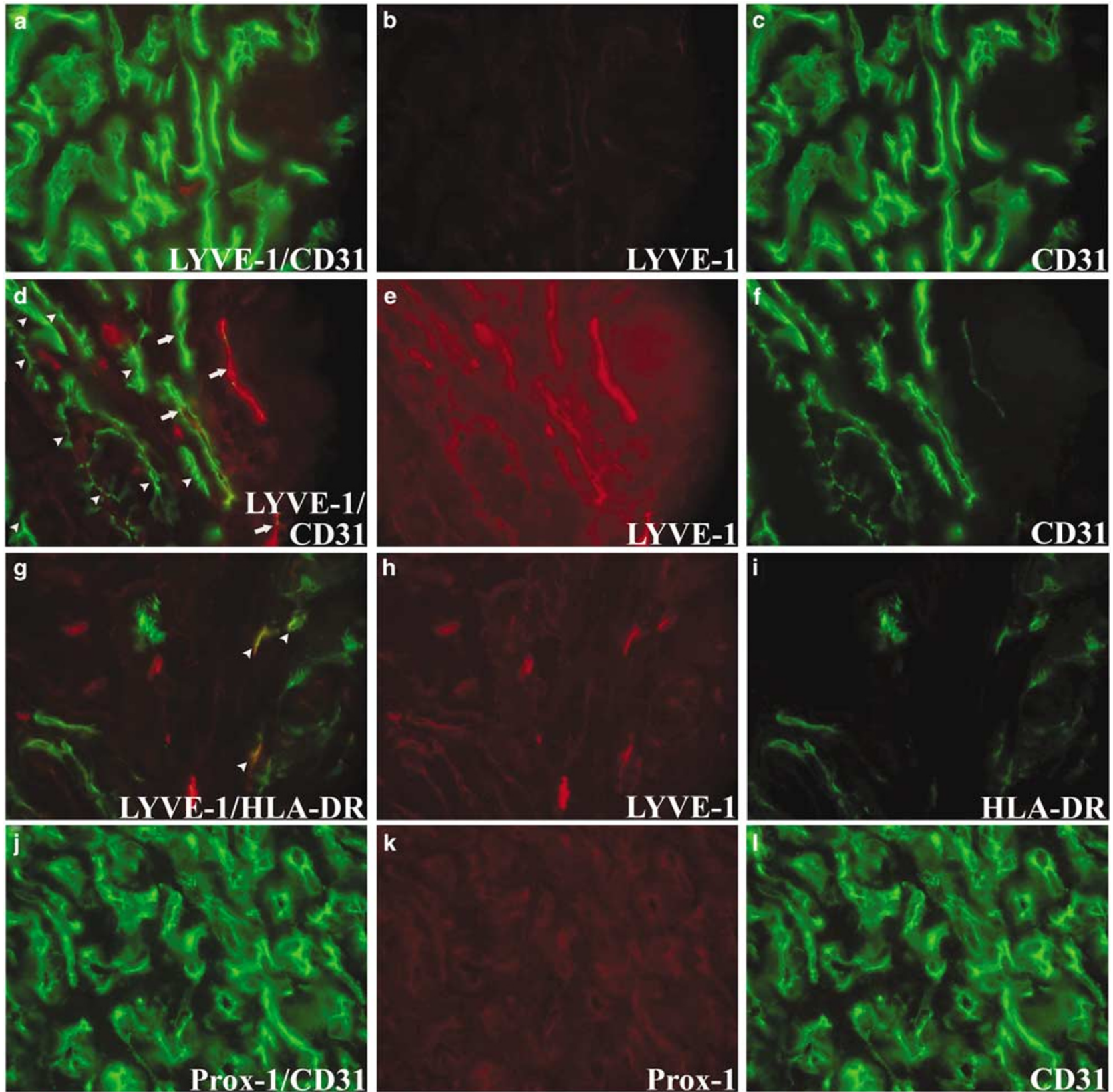

k
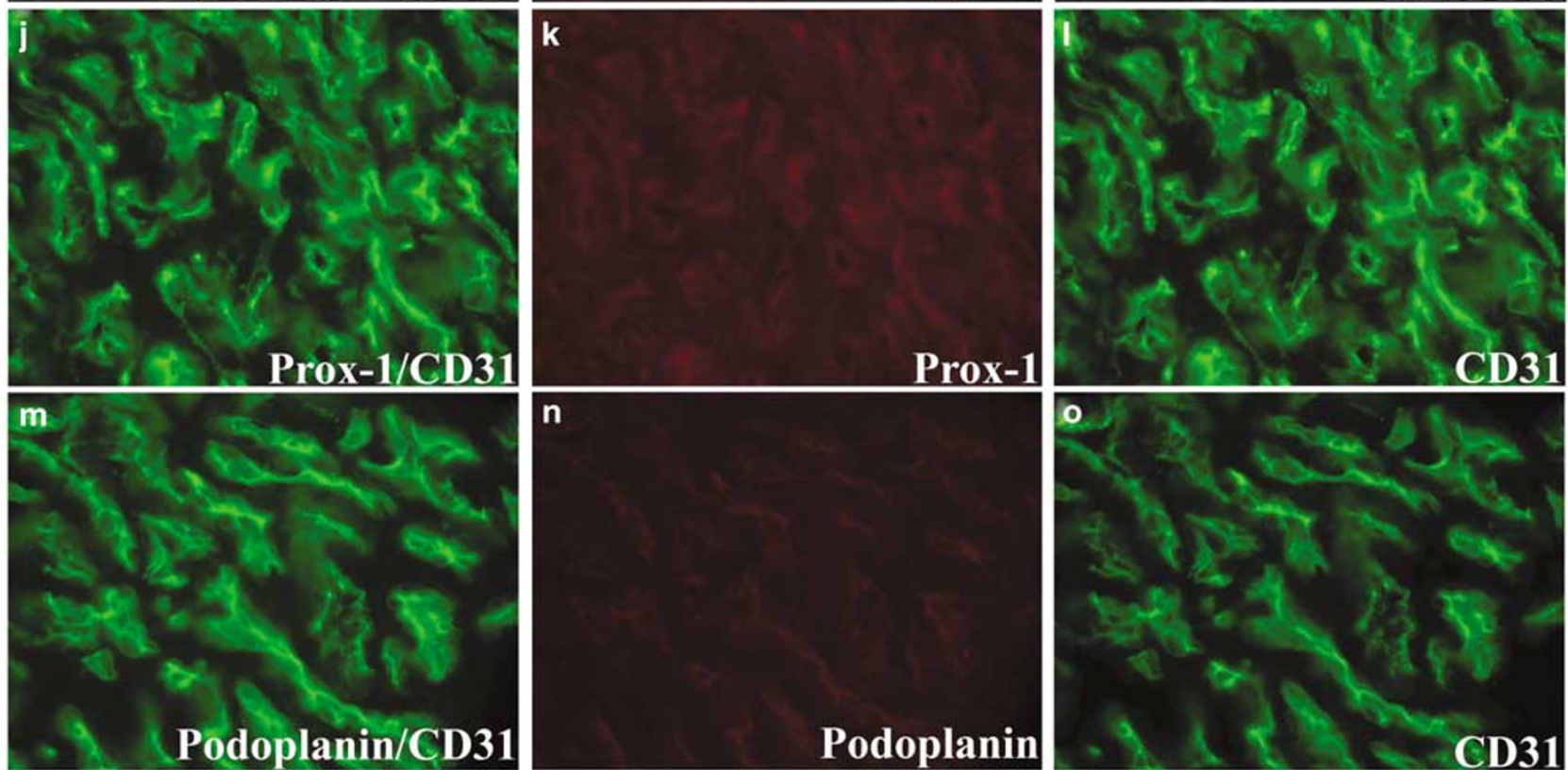

Prox-1

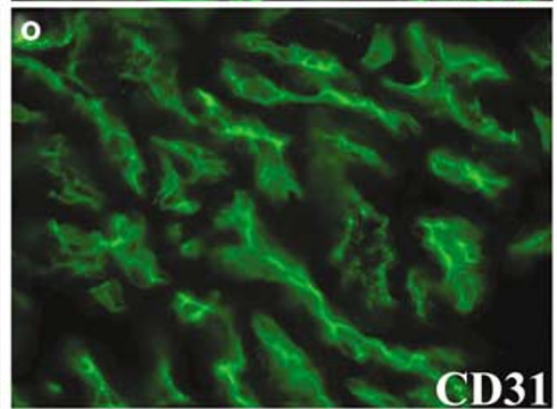

Figure 2 Double immunofluorescence staining of proliferating infantile hemangioma tissue. (a), (b) and (c) Double immunofluorescence staining for LYVE-1 (red) and CD31 (green) demonstrates no overlap (yellow) in hemangioma endothelial cells. (d), (e) and (f) Intraseptal lymphatic vessels, however, coexpress LYVE-1 and CD31 (arrows). Intraseptal blood vessels, in turn, are solely CD31 positive, but LYVE1 negative (arrowheads). (g), (h) and (i) Only scattered LYVE-1 ${ }^{+}$cells are seen in the dense network of surrounding HLA-DR ${ }^{+}$cells with dendritic cell morphology (arrowheads). (j), (k), (l), (m), (n) and (o) Merged images for Prox-1 and CD31 reveal absence of Prox-1 in lesional $\mathrm{CD}_{3}{ }^{+}$endothelial cells. Similar results are obtained in double staining for podoplanin and CD31. Original magnifications: $\times 400(\mathbf{a}-\mathbf{o})$. 
CD206/DC-SIGN were present, whereas CD1a, CD208/DC-LAMP and CD303/BDCA-2 were absent (data not shown). This pattern of reactivity suggests a close relationship to monocyte/macrophage or dendritic cell lineages, although excluding epidermal Langerhans cells, plasmacytoid and mature dendritic cells of any type.

To assess the specificity and sensitivity of the lymphatic endothelial cell markers used, their distribution on endothelial cells of lymphangioma tissue was evaluated in serial sections showing mutually exclusive expression of LYVE-1, Prox-1, podoplanin and D2-40. Importantly, in comparison to infantile hemangiomas, no adjacent HLA-DRpositive cells around vascular structures were found in lymphangioma tissue (data not shown).

\section{Discussion}

Infantile hemangiomas are common benign vascular tumors, distinctive for their characteristically predictable biologic behavior: They generally appear soon after birth, proliferate rapidly during the first year of life and slowly involute over a period of several years. ${ }^{3}$ Despite intensive scrutiny, the mechanisms that orchestrate growth and regression of hemangioma vessels remain obscure. A number of distinct hypotheses have been put forth to account for hemangiogenesis, including genetic predisposition, imbalance of angiogenic factors, dysregulated vasculogenesis, clonality and placental origin of hemangioma endothelial cells. ${ }^{4-9}$ Owing to the exclusive expression of LYVE-1 by proliferating hemangioma endothelial cells, recent studies suggested an intrinsic defect in hemangioma endothelial cell maturation. ${ }^{11}$ This prompted us to study the expression of the lymphatic endothelial cell-specific markers LYVE-1, Prox-1, podoplanin and D2-40 in a large number of infantile hemangiomas the growth status of which was categorized to a part by the staining with $\beta 4$ integrin. The lymphatic endothelial hyaluronan receptor LYVE-1 is considered as a marker for normal and tumor-associated lymphatic vessels and Prox-1 is a homeobox gene product that controls the development of lymphatic progenitors from embryonic veins, whereas podoplanin, a transmembrane mucoprotein, plays an important role in the migration, adhesion and tube formation of lymphatic endothelial cells. ${ }^{13-15}$ Recently, D2-40 has been identified as a specific antibody against human podoplanin. ${ }^{16,17}$

Unexpectedly, LYVE-1 expression was consistently absent in immunohistochemical and double immunofluorescence stainings of both $\beta 4$-negative proliferating and $\beta 4$-positive involuting infantile hemangioma samples (Figures 1a and 2a-c), except for one case. This observation is in sharp contrast to the report by Dadras et al, ${ }^{11}$ who demonstrated exclusive expression of LYVE-1 in endothelial cells of proliferating hemangioma vessels and its dis- appearance during involution, but without specifying the criteria for the proliferative and involutional phases. Hitherto, there are no internationally agreed criteria for proliferating and involuting infantile hemangiomas. According to the current understanding, the proliferative phase is histologically hallmarked by endothelial and pericytic hyperplasia, whereas the involutional phase is predominated by apoptosis and fibrosis, clinically corresponding to slight atrophic skin changes. ${ }^{3}$ To help to distinguish the growth phases of infantile hemangiomas at the cellular level, several biomolecular markers have been introduced, including $\beta 4$ integrin by our group. ${ }^{4,18,19}$ Specifically, we found expression of $\beta 4$ integrin only in involuting infantile hemangiomas. This led us to suggest an antiangiogenic effect of $\beta 4$ integrin with induction of arrest of endothelial cell proliferation, presumably via integrin-mediated signal transduction pathways.

Admittedly, we do not know why our results are distinct from the study by Dadras et al. Similar to their study, our samples were diagnosed as infantile hemangioma by characteristic clinical and histological features. Moreover, sex, age and site of distribution in our series of infantile hemangiomas were similar to those provided by Dadras et al, implying that our proliferating hemangioma samples did not derive from more full-grown children and were thus not involuting.

One possible explanation for the discrepant findings could be the variability of immunohistochemical and immunofluorescent methods used. However, the technical aspects unlikely explain the seen discrepancies because peritumoral and intraseptal lymphatic vessels as well as lymphangioma tissue were stained brightly with LYVE-1. Also, the LYVE-1 antibody used in this and the previous study was identical.

As shown by Dadras et al, not all proliferating hemangioma vessels expressed LYVE-1, indicating that expression of LYVE-1 in infantile hemangiomas is not a universal phenomenon. Most cases displayed less than 10\% LYVE-1-positive hemangioma vessels, and only a minor subset more than $50 \% .{ }^{11}$ Based on these findings, we suppose that variants of infantile hemangiomas with different LYVE-1 expression patterns exist. It is conceivable that we examined in our study primarily those infantile hemangiomas with less or without LYVE-1 expression, as only one case showed LYVE-1 staining in the majority $(<50 \%)$ of proliferating hemangioma vessels.

In accordance with Dadras et al, we detected LYVE-1- and HLA-DR-positive perivascular cells in proliferating as well as involuting infantile hemangiomas, albeit of only some patients (Figures $1 \mathrm{~d}$ and $2 \mathrm{~g}-\mathrm{i}$ ). As described earlier in detail, the staining pattern of these cells, that is, HLA-DR, CD68 and DC-SIGN positive, CD1a, DC-LAMP and BDCA-2 negative, is most compatible with monocytes/ 
macrophages or dendritic cells. ${ }^{4}$ At the present time, we do not know their function. Evidently, their presence alone does not imply that they are involved in growth or regression of infantile hemangiomas because they were found during both proliferative and involutional phases. We rather speculate that they might influence vascular formation by secreting angiogenic or antiangiogenic cytokines/chemokines. Of note, in lymphangioma tissue, there were no HLA-DR-positive cells around vascular structures. This strengthens our hypothesis that hemangioma endothelial cells and the surrounding hematopoietic cells might originate from a common precursor and that vasculogenesis might be the underlying pathogenetic mechanism in infantile hemangioma.

Immunostains revealed no expression of Prox-1, podoplanin and D2-40 of lesional capillaries at all stages (Figures 1c, e-h and 2j-o), although lymphatic vessels at the tumor margin and in the tumor septum did react with these lymphatic endothelial cell-specific markers (Figures 1a, c, e-h and 2d-f). As human podoplanin was shown to be specifically recognized by the D2-40 antibody, ${ }^{17}$ staining with the D2-40 antibody can be regarded as an internal control for the podoplanin antibody. Results obtained here are consistent with the work of others. Neither Dadras et $a l^{11}$ nor Wilting et $a l^{20}$ observed Prox-1-positive hemangioma capillaries. Conversely. Prox-1 was found in the nucleus of lymphatic capillaries adjacent to hemangioma lobules. Galambos and Nodit ${ }^{21}$ noted no staining of D2-40 in endothelial cells of infantile hemangiomas. Independent confirmation has also been provided by Fukunaga. ${ }^{22}$ The fact that infantile hemangiomas are devoid of lymphatic endothelial cell-specific markers underscores their blood endothelial cell origin, evidently without acquiring lymphatic competence.

In summary, the data presented here differ from those reported by Dadras et al. In contrast to Dadras et al, we could not document a significant difference in the expression of LYVE-1 during the proliferative and involutional phases. Accordingly, LYVE-1 does not seem to be a specific marker for proliferating infantile hemangiomas. We speculate that variants of infantile hemangiomas with a distinct LYVE-1 expression pattern contributed to these discrepancies.

Furthermore, the lack of LYVE-1 expression contradicts the hypothesis raised by Dadras et al that proliferating infantile hemangiomas are arrested in an early stage of vascular development due to their immature LYVE-1-positive phenotype that resembles the embryogenic cardinal vein. ${ }^{11,12}$ Our study clearly illustrates that this suggested intrinsic defect in endothelial cell maturation is not an explanation for the excessive endothelial cell proliferation seen postpartum.

In conclusion, despite their frequency, the pathogenesis of infantile hemangiomas remains a mystery that poses a challenge to be disclosed.

\section{References}

1 Drolet BQ, Esterly NB, Frieden IL. Hemangiomas in children. N Engl J Med 1999;341:173-181.

2 Enjolras JB, Mulliken JB. Vascular tumors and vascular malformations (new issues). Adv Dermatol 1997;13: 375-422.

3 Mulliken JB, Glowacki J. Hemangiomas and vascular malforamtions in infants and children: a classification based on endothelial characteristics. Plast Reconstr Surg 1982;69:412-422.

4 Nguyen VA, Fürhapter C, Romani N, et al. Infantile hemangioma is a proliferation of $\beta 4$-negative endothelial cells adjacent to HLA-DR ${ }^{+}$cells with dendritic cell morphology. Hum Pathol 2004;35: 739-744.

5 Marchuk DA. Pathogenesis of hemangioma. J Clin Invest 2001;107:665-666.

6 Bielenberg DR, Bucana CD, Sanchez R, et al. Progressive growth of infantile cutaneous hemangiomas is directly correlated with hyperplasia and angiogenesis of adjacent epidermis and inversely correlated with expression of endogenous angiogenesis inhibitor, IFNbeta. Int J Oncol 1999;14:401-408.

7 North PE, Wanner M, Mizeracki A, et al. A unique microvascular phenotype shared by juvenile hemangiomas and human placenta. Arch Dermatol 2001;137: 559-570.

8 Boye E, Yu Y, Paranya G, et al. Clonality and altered behavior of endothelial cells from hemangiomas. J Clin Invest 2001;107:745-752.

9 Walter JW, North PE, Waner M, et al. Somatic mutation of vascular endothelial growth factor receptors in juvenile hemangioma. Genes Chromosomes Cancer 2002;33:295-303.

10 Carmeliet P, Jain RK. Angiogenesis in cancer and other diseases. Nature 2000;407:249-257.

11 Dadras SS, North PE, Bertoncini J, et al. Infantile hemangiomas are arrested in an early developmental vascular differentiation state. Mod Pathol 2004;17: 1068-1079.

12 Oliver G, Detmar M. The rediscovery of the lymphatic system: old and new insights into the development and biological function of the lymphatic vasculature. Genes Dev 2002;16:773-783.

13 Banerji S, Ni J, Wang SX, et al. LYVE-1, a new homologue of the CD44 glycoprotein, is a lymphspecific receptor for hyaluronan. J Cell Biol 1999;144: 789-801.

14 Breiteneder-Geleff S, Soleiman A, Kowalski H, et al. Angiosarcomas express mixed endothelial phenotypes of blood and lymphatic capillaries: podoplanin as a specific marker for lymphatic endothelium. Am J Pathol 1999;154:385-394.

15 Wigle JT, Harvey N, Detmar M, et al. An essential role for Prox1 in the induction of the lymphatic endothelial cell phenotype. EMBO J 2002;21: 1505-1513.

16 Kahn HJ, Marks A. Monoclonal antibody D2-40, a new marker of lymphatic endothelium, reacts with Kaposi's sarcoma and a subset of angiosarcomas. Mod Pathol 2002;15:434-440.

17 Schacht V, Dadras SS, Johnson LA, et al. Up-regulation of the lymphatic marker podoplanin, a mucin-type transmembrane glycoprotein, in human squamous cell carcinomas and germ cell tumors. Am J Pathol 2005;166:913-921. 
18 Takahashi K, Mulliken JB, Kozakewich HP, et al. Cellular markers that distinguish the phases of hemangioma during infancy and childhood. J Clin Invest 1994;93:2357-2364.

19 Frischer JS, Huang J, Serur A, et al. Biomolecular markers and involution of hemangiomas. J Pediatr Surg 2004;39:400-404.

20 Wilting J, Papoutsi M, Christ B, et al. The transcription factor Prox1 is a marker for lymphatic endothelial cells in normal and diseased human tissues. FASEB J 2002;16:1271-1273.

21 Galambos C, Nodit L. Identification of lymphatic endothelium in pediatric vascular tumors and malformations. Pediatr Dev Pathol 2005;8: 181-189.

22 Fukunaga M. Expression of D2-40 in lymphatic endothelium of normal tissues and in vascular tumours. Histopathology 2005;46:396-402. 